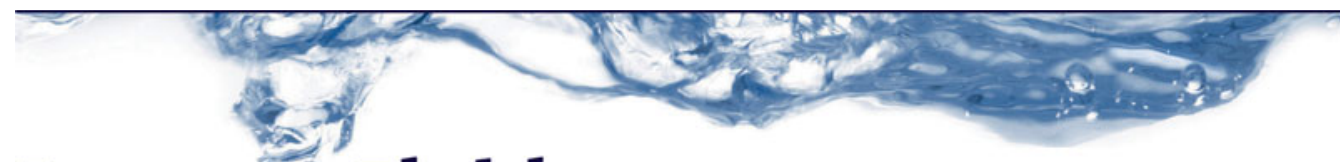

Focus on Fluids

journals.cambridge.org/focus

\title{
The organizing centre for the flow around rapidly spinning cylinders
}

\section{Morten Brøns †}

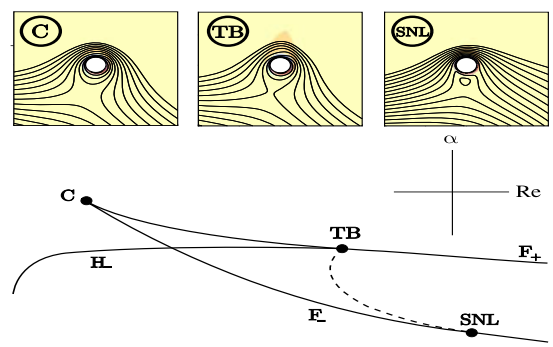

Department of Applied Mathematics and Computer Science,

Technical University of Denmark,

2800 Kongens Lyngby, Denmark

The flow around a rotating circular cylinder has a parameter regime with a complex pattern of periodic solutions and multiple steady states. Sierra et al. (J. Fluid Mech., vol. 905, 2020, A2) provide a complete bifurcation analysis of this regime. The numerical computations are guided by a qualitative analysis of the bifurcations stemming from a highly degenerate singular dynamical system. Surprisingly, the dynamics of the singular system itself cannot be realized as a specific flow, but acts mathematically as an organizer of the physical bifurcation diagram.

Key words: wakes, bifurcation, instability

\section{Introduction}

On a list of the most studied fluid mechanics problems, the flow around a circular cylinder would surely be at the top end. The cylinder is the prototype bluff body, and the flow around it has been used to gain insight into separated flows, their stability and ways to control them.

The flow in an unbounded domain depends on a single dimensionless parameter, the Reynolds number $R e=U D / v$, where $U$ is the incoming fluid velocity, $D$ is the diameter of the cylinder and $v$ is the kinematic viscosity of the fluid. The most fundamental instability occurs at $R e_{H} \approx 46$, where the symmetric steady flow becomes unstable, and a periodic flow appears (Williamson 1996). The periodic flow leads to vortex shedding, giving rise to the famous Kármán vortex street where vortices of alternate signs are shed from the cylinder. In terms of bifurcation theory, the transition is a supercritical Hopf bifurcation (Provansal, Mathis \& Boyer 1987).

If the cylinder also rotates, another dimensionless parameter appears, $\alpha=\Omega D / 2 U$, where $\Omega$ is the angular velocity of the cylinder. The rotation breaks the symmetry of 


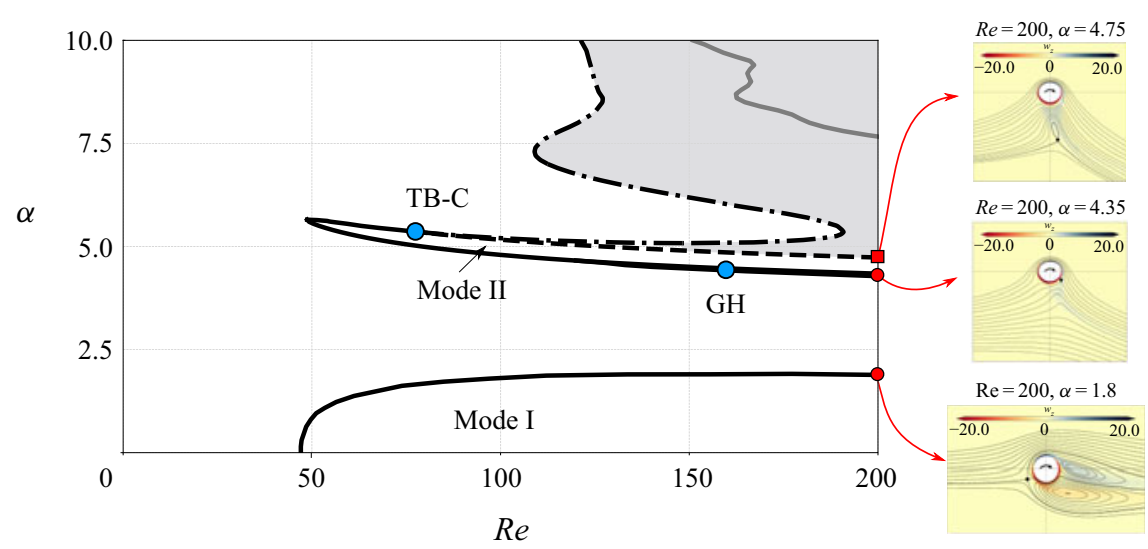

FIGURE 1. Bifurcations diagram showing curves of elementary bifurcations in the $(R e, \alpha)$ parameter plane. Boxes to the right show streamlines and vorticity at the bifurcation points for $R e=200$. Full lines denote Hopf bifurcations, dashed and dash-dotted lines are fold bifurcations. There are three steady states in the grey region. Mode I is the periodic Kármán wake; mode II is the low-frequency periodic flow; TB-C marks a Takens-Bogdanov point and a cusp that are very close; $\mathrm{GH}$ is a generalized Hopf point which will not be discussed here. From Sierra et al. (2020).

the steady solution and stabilizes it, such that the critical Reynolds number $R e_{H}$ increases rapidly with $\alpha$ in the range $0<\alpha<2$, see figure 1 .

For higher values of $\alpha$ a more complex scenario occurs. Low-frequency periodic solutions (denoted mode II) with shedding of same-sign vortices have been found (Stojković, Breuer \& Durst 2002), as well as a range with up to three steady states (Pralits, Brandt \& Gianetti 2010; Rao et al. 2013).

Sierra et al. (2020) give for the first time a complete description of the intricate pattern of bifurcations that connect these states under variation of $R e$ and $\alpha$. The important insight is that the bifurcation diagram can be understood from an organizing centre, a state with highly degenerate dynamics. The organizing centre has codimension three, meaning that three parameters are needed to realize all possible kinds of dynamics near it. This is surprising, as the system at hand only has two parameters, and the dynamics of the organizing centre cannot be realized for the physical system. Nevertheless, this 'super' organizing centre is a most useful mathematical abstraction which Sierra et al. (2020) use as a guide to conjecture the structure of the physical bifurcation diagram and verify it by numerical simulations.

\section{Overview}

A two-parameter bifurcation diagram is built up by a number of curves of elementary bifurcations. For the rotating cylinder, curves of Hopf bifurcations and fold (saddle-node) bifurcations occur. In a fold bifurcation two steady states, one stable, one unstable, appear out of the blue. The bifurcation curves can meet in special singularities, codimension-two points, also denoted organizing centres (Golubitsky \& Schaeffer 1985). By analysing simple representative cases, normal forms, the qualitative structure of the bifurcation diagram near a codimension-two singularity can be found (Kuznetsov 2004). 

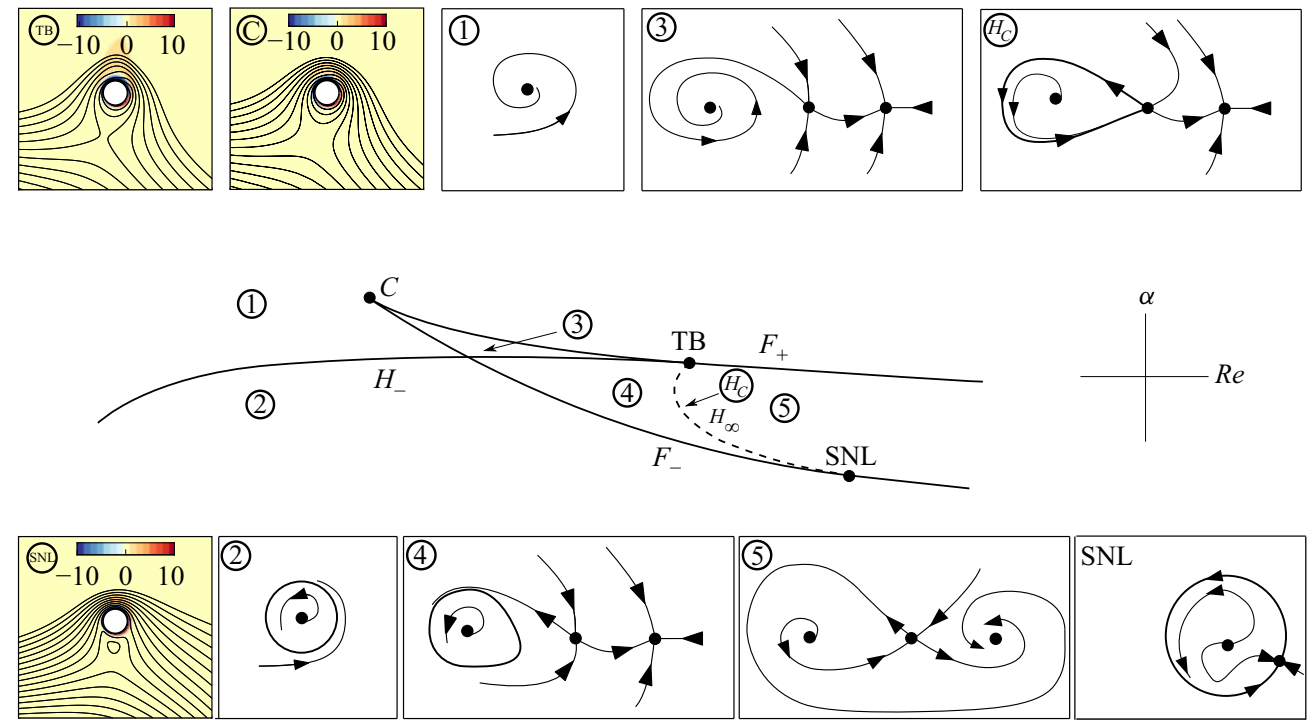

FIGURE 2. A two-dimensional slice through the three-dimensional bifurcation diagram of the degenerate Takens-Bogdanov bifurcation. Here $C$, cusp bifurcation; TB, Takens-Bogdanov point; SNL, saddle-node-loop point; $H_{-}$, Hopf bifurcation curve; $F_{+}, F_{-}$, fold bifurcation curves; $H_{\infty}$, homoclinic bifurcation curve. Typical dynamics in each sector are shown in the boxes. Boxes with yellow background show streamlines and vorticity at the codimension-two points. From Sierra et al. (2020).

Sierra et al. (2020) argue that two codimension-two singularities are relevant for the rotating cylinder. One is the cusp, where two fold curves meet. Close to a cusp up to three steady states can be present. The other is the Takens-Bogdanov (TB) point, where a Hopf curve and a fold curve meet. From a TB point a curve of homoclinic bifurcations also emanates. In these bifurcations a periodic state disappears as the frequency tends to zero. This fits well with the low-frequency mode II periodic flows. Neither the cusp nor the TB point alone can account for all the available observations. Both will be needed for a complete description.

Figure 1 show the curves of elementary bifurcations computed by Sierra et al. (2020). As anticipated, both a cusp and a TB point are found. The two points are very close, $(R e, \alpha)=(75.6,5.38)$ and $(77.6,5.36)$, respectively. This indicates that a small change of the dynamical system at hand could make the two points merge into a more degenerate singularity. Such a degenerate TB point has codimension three and the corresponding local bifurcation diagram has three parameters. The bifurcation diagram has been determined for a normal form by Dumortier et al. (1991), and it is expected that the bifurcation diagram for the rotating cylinder will correspond to a two-dimensional slice in the extended three-dimensional parameter space. A candidate for such a slice is shown in figure 2 . As required, it includes both a cusp and a TB point, but there is an additional organizing centre, a saddle-node loop. At this point the dynamics exhibits a fold bifurcation point on a limit cycle, and in the bifurcation diagram a curve of homoclinic bifurcations emanates which ends at the TB point. By careful simulations, Sierra et al. (2020) confirm that all the features of figure 2 are indeed present for the rotating cylinder. However, on the scale of figure 1 the details of figure 2 are too fine to be seen. Hence, the organizing centres and the bifurcation curves joining them in the two-dimensional 
$(R e, \alpha)$ plane are themselves organized from a 'super' organizing centre, the degenerate TB point.

\section{Future}

The question naturally arises if the setting of the problem could be modified such that a third parameter appears, and the degenerate TB point could be realized physically. Sierra et al. (2020) suggest considering compressibility, or changing the external boundary conditions by confinement or shear in the incoming flow. If such attempts were successful, the full three-parameter diagram of the degenerate TB bifurcation could be realized, and bifurcation diagrams different from figure 2, corresponding to different slices in the three-dimensional parameter space, would occur. If the third parameter is sufficiently large, the bifurcation diagram in figure 2 could occupy a substantially larger part of the $(R e, \alpha)$ space and be experimentally observable.

It would be interesting to understand better why the flow around a rotating cylinder is so close to a codimension-three singularity. The rotating cylinder is unique in the sense that the boundary is time independent, and steady flows are allowed. This is not the case for any other rotating shape. Is there a hidden symmetry?

The approach of Sierra et al. (2020) will be useful in other similar problems. What is required is a problem that is relatively simple from a dynamical point of view, that is, only steady states and periodic flows occur, but which has complex dependence on parameters. Many two-dimensional flows at low $R e$ will fit in this category.

\section{Declaration of interest}

The author reports no conflict of interest.

\section{References}

Dumortier, F., Roussarie, R., Sotomayor, J. \& Zoladec, H. 1991 Bifurcations of Planar Vector Fields: Nilpotent Singularities and Abelian Integrals. Lecture Notes in Mathematics, vol. 1480. Springer.

Golubitsky, M. \& SchaefFer, D. G. 1985 Singularities and Groups in Bifurcation Theory I. Applied Mathematical Sciences, vol. 51. Springer.

Kuznetsov, Y. A. 2004 Elements of Applied Bifurcation Theory, 3rd edn. Applied Mathematical Sciences, vol. 112. Springer.

PRALITS, J. O., BRANDT, L. \& GiAnetTi, F. 2010 Instability and sensitivity of the flow around a rotating circular cylinder. J. Fluid Mech. 650, 513-536.

Provansal, M., Mathis, C. \& Boyer, L. 1987 Bénard-von Kármán instability: transient and forced regimes. J. Fluid Mech. 182, 1-22.

Rao, A., Leontini, J. S., Thompson, M. C. \& Hourigan, K. 2013 Three-dimensionality in the wake of a rapidly rotating cylinder in uniform flow. J. Fluid Mech. 730, 379-391.

Sierra, J., Fabre, D., Citro, V. \& Giannetti, F. 2020 Bifurcation scenario in the two-dimensional laminar flow past a rotating cylinder. J. Fluid Mech. 905, A2.

Stojković, D., BREuer, M. \& DURST, F. 2002 Effect of high rotation rates on the laminar flow around a circular cylinder. Phys. Fluids 14 (9), 3160-3178.

Williamson, C. H. K. 1996 Vortex dynamics in the cylinder wake. Annu. Rev. Fluid Mech. 28 (1), 477-539. 